\title{
ON THE PROBABILITY OF THE EVENT: IN $n$ GENERALIZED ALLOCATION SCHEMES THE VOLUME OF EACH CELL DOES NOT EXCEED $r$.
}

\author{
A.I. AFONINA, I.R. KAYUMOV, A.N. CHUPRUNOV
}

\begin{abstract}
We consider $n$ identical generalized schemes of allocating particles in cells. We study the probability of the event: for each generalized allocation scheme, there are at most $r$ particles in each cell, where $r$ is a given number. We obtain an asymptotic estimate for this probability and we consider the application of the obtained results to an antinoise coding.
\end{abstract}

Keywords: generalized allocation scheme, Cauchy integral, Hamming code.

Mathematics Subject Classification: 60K30

\section{INTRODUCTION AND MAIN RESULTS}

Let $\xi, \xi_{j}, 1 \leqslant j \leqslant N$, be independent identically distributed integer-valued random variables. We recall [1] that random variables $\eta_{1}, \ldots, \eta_{\mathrm{N}}$ are called a generalized scheme of allocating $m$ particles in $N$ cells if their joint distribution is of the form

$$
\mathbf{P}\left\{\eta_{1}=k_{1}, \ldots, \eta_{\mathrm{N}}=k_{N}\right\}=\mathbf{P}\left\{\xi_{1}=k_{1}, \ldots, \xi_{N}=k_{N} \mid \xi_{1}+\cdots+\xi_{N}=m\right\},
$$

where $k_{1}+k_{2}+\cdots+k_{N}=m$.

Many allocating schemes of the discrete probability theory such as the scheme of allocating discernible particles in cells, the scheme of allocating indiscernible particles in cells, random permutations, random forest are generalized allocating schemes (for generalized allocating schemes see [2]-6]).

In what follows, as random variables $\xi, \xi_{j}$, we shall consider random variables $\xi=\xi(x), \xi_{j}=$ $\xi_{j}(x), x>0$, with the distribution

$$
\mathbf{P}(\xi(x)=k)=\frac{a_{k} x^{k}}{S(x)}, \quad k=0,1,2 \ldots,
$$

where $S(x)=\sum_{k=0}^{\infty} a_{k} x^{k}$ is the sum of a series with non-negative coefficients having a positive convergence radius $R$. In this case we shall say that generalized allocating scheme $\eta_{1}, \ldots, \eta_{\mathrm{N}}$ is defined by function $S(x)$.

Random variables $\xi=\xi(x), \xi_{j}=\xi_{j}(x)$ were introduced in work [7]. In works [7]-[9], there were obtained limiting theorems for the sums of random variables $\xi_{j}(x)$.

We consider the event $A_{N}(m, r)$ that in a generalized scheme of allocating $m$ particles in $N$ cells each cell contains at most $r$ particles:

$$
A_{N}(m, r)=\left\{\omega \in \Omega: \eta_{1}(\omega) \leqslant r, \ldots, \eta_{\mathrm{N}}(\omega) \leqslant r\right\}=\left\{\omega \in \Omega: \max _{1 \leqslant i \leqslant N} \eta_{\mathrm{i}}(\omega) \leqslant r\right\} .
$$

The probability of event $A_{N}(m, r)$ has the following representation.

A.I. Afonina, I.R. Kayumov, A.N. Chuprunov, On the Probability of the Event: in $n$ GenerALIZED ALLOCATION SCHEMES THE VOLUME OF EACH CELL DOES NOT EXCEED $r$.

(C) Afonina A.I., Kayumov I.R., Chuprunov A.N. 2016.

Submitted December 29, 2015. 
Lemma A. Let $S_{r}(z)=\sum_{k=0}^{r} a_{k} z^{k}$ be a partial sum of series $S(z), a_{m}\left(S^{N}\right)$ be the $m$-th coefficient in the expansion of function $(S(z))^{N}$, which is the $N$-th power of function $S(z)$, $a_{m}\left(S_{r}^{N}\right)$ be the $m$-th coefficient in the expansion of function $\left(S_{r}(z)\right)^{N}$, which is the $N$-th power of function. Then

$$
\mathbf{P}\left(A_{N}(m, r)\right)=\frac{a_{m}\left(S_{r}^{N}\right)}{a_{m}\left(S^{N}\right)}=\frac{\frac{1}{2 \pi \mathrm{i}} \oint_{C} \frac{S_{r}(z)^{N}}{z^{m+1}} d z}{\frac{1}{2 \pi \mathrm{i}} \oint_{C} \frac{S(z)^{N}}{z^{m+1}} d z},
$$

where $C$ is a closed contour passed in the positive direction whose interior contains no zeroes of functions $S$ and $S_{r}$.

Let $\eta_{\mathrm{i} 1}, \ldots, \eta_{\mathrm{iN}}, 1 \leqslant i \leqslant n$, be the sequence of independent identically distributed generalized schemes of allocating $m$ particles in $N$ cells, that is, such that the joint distribution is given by the formula

$$
\mathbf{P}\left\{\eta_{\mathrm{i} 1}=k_{1}, \ldots, \eta_{\mathrm{iN}}=k_{N}\right\}=\mathbf{P}\left\{\xi_{1}=k_{1}, \ldots, \xi_{N}=k_{N} \mid \xi_{1}+\cdots+\xi_{N}=m\right\},
$$

where $k_{1}+k_{2}+\cdots+k_{N}=m$.

We shall assume that condition $\left(A_{k}\right)$ is satisfied: $a_{0}=\cdots=a_{k-1}=0, a_{k}=1, a_{k+1}>0$.

Employing representation (1), in [13] we proved the following theorem.

Theorem B. Assume that condition $\left(A_{0}\right)$ is satisfied and $m>r$. Then

$$
\mathbf{P}\left(A_{n, N}(m, r)\right)=\exp \left[\frac{-m(m-1) \cdots(m-r)}{N^{r-1}} \frac{a_{r+1}}{a_{1}^{r+1}} \alpha\left(1+O\left(\frac{1}{N}\right)\right)\right]
$$

as $n, N \rightarrow \infty$ so that $\alpha=o\left(N^{r}\right)$, where $\alpha$ the ratio of the amount of independent series of particles and the amount of cells, i.e.,

$$
\alpha=\alpha_{n N}=\frac{n}{N} .
$$

In particular, if $m>r, n, N \rightarrow \infty$ so that $\frac{n}{N^{r}} \rightarrow \beta$, where $\beta<\infty$, then

$$
\mathbf{P}\left(A_{n, N}(m, r)\right) \rightarrow \exp \left[-m(m-1) \cdots(m-r) \frac{a_{r+1}}{a_{1}^{r+1}} \beta\right] .
$$

The following generalization of Theorem B for arbitrary $k$ is true.

Theorem 1. Assume that condition $\left(A_{k}\right)$ is satisfied and $k<r<K<\infty$. We denote $m_{1}=m-k N$. Then

$$
\mathbf{P}\left(A_{n, N}(m, r)\right)=\exp \left[\frac{-m_{1}\left(m_{1}-1\right) \cdots\left(m_{1}-(r-k)\right)}{N^{r-k-1}} \frac{a_{r+1}}{a_{k+1}^{r-k+1}} \alpha\left(1+O\left(\frac{1}{N}\right)\right)\right],
$$

uniformly in $m_{1} \in(k, K]$ as $m, n, N \rightarrow \infty$ so that $\alpha=o\left(N^{r-k}\right)$. In particular, if $m, n, N \rightarrow$ $\infty$ so that $m_{1}$ is fixed, $m_{1}>r$ and $\frac{n}{N^{r-k}} \rightarrow \beta$, where $\beta<\infty$, then

$$
\mathbf{P}\left(A_{n, N}(m, r)\right) \rightarrow \exp \left[-m_{1}\left(m_{1}-1\right) \cdots\left(m_{1}-(r-k)\right) \frac{a_{r+1}}{a_{k+1}^{r-k+1}} \beta\right] .
$$

Let us consider the generalized allocating scheme $\eta_{1}^{*}, \ldots, \eta_{\mathrm{N}}^{*}$ defined by the function $S^{*}(x)=$ $\sum_{i=0}^{\infty} a_{i}^{*} x^{i}$ and the event $A_{n, N}^{*}(m, r)=\cap_{i=1}^{n}\left\{\eta_{\mathrm{i} 1}^{*} \leqslant r, \ldots, \eta_{\mathrm{iN}}^{*} \leqslant r\right\}$, where $\eta_{\mathrm{i} 1}, \ldots, \eta_{\mathrm{iN}}, 1 \leqslant i \leqslant n$, are independent copies of scheme $\eta_{1}^{*}, \ldots, \eta_{\mathrm{N}}^{*}$. 
Corollary. Assume that condition $\left(A_{k}\right)$ is satisfied for schemes $\eta_{1}, \ldots, \eta_{\mathrm{N}}$ and $\eta_{1}^{*}, \ldots, \eta_{\mathrm{N}}^{*}$, $a_{k+1}=a_{k+1}^{*}, a_{r-k+1}=a_{r-k+1}^{*}, k<r<K<\infty$. Then

$$
\mathbf{P}\left(A_{n, N}(m, r)\right)=\mathbf{P}\left(A_{n, N}^{*}(m, r)\right)^{\left(1+O\left(\frac{1}{N}\right)\right)}
$$

uniformly in $m_{1} \in(k, K]$ as $m, n, N \rightarrow \infty$ so that $\alpha=o\left(N^{r-k}\right)$.

To prove the corollary, it is sufficient to note that the probabilities $\mathbf{P}\left(A_{n, N}(m, r)\right)$ and $\mathbf{P}\left(A_{n, N}^{*}(m, r)\right)$ satisfy formula (3).

Remark 1. The random variable $\eta_{(N)}=\max _{1 \leqslant i \leqslant N} \eta_{i}$ is called the maximal volume of a cell. Many works were devoted to studying the limiting behavior of the maximal volume of a cell [2], 6], [17]. As $n=1$, Theorem $B$ and Theorem 1 can be regarded as the limiting theorem for the distribution function of random variable $\eta_{(N)}$ in the case, when $m_{1}$ is bounded and $N \rightarrow \infty$.

Let $0 \leqslant r_{1}<r_{2} \leqslant \infty$. Consider random variables $\eta_{1}^{\prime}, \ldots, \eta_{\mathrm{N}}^{\prime}$ having the joint distribution

$$
\mathbf{P}\left\{\eta_{1}^{\prime}=k_{1}, \ldots, \eta_{\mathrm{N}}^{\prime}=k_{N}\right\}=\mathbf{P}\left\{\eta_{1}=k_{1}, \ldots, \eta_{\mathrm{N}}=k_{N} \mid r_{1} \leqslant \min _{1 \leqslant i \leqslant N} \eta_{i}, \max _{1 \leqslant i \leqslant N} \eta_{i} \leqslant r_{2}\right\},
$$

where $k_{1}+k_{2}+\cdots+k_{N}=m, r_{1} \leqslant k_{i} \leqslant r_{2}, 1 \leqslant i \leqslant N$.

Theorem 2. Random variables $\eta_{1}, \ldots, \eta_{\mathrm{N}}^{\prime}$ are generalized scheme of allocating $m$ particles in $N$ cells defined by the function $S_{r_{1} r_{2}}(x)=\sum_{i=r_{1}}^{r_{2}} a_{i} x^{i}$.

Let $0<r_{2} \leqslant \infty$. Consider random variables $\eta_{1}^{\{2\}}, \ldots, \eta_{\mathrm{N}}^{\{2\}}$ with joint distribution

$$
\mathbf{P}\left\{\eta_{1}^{\{2\}}=k_{1}, \ldots, \eta_{\mathrm{N}}^{\{2\}}=k_{N}\right\}=\mathbf{P}\left\{\eta_{1}=k_{1}, \ldots, \eta_{\mathrm{N}}=k_{N} \mid \max _{1 \leqslant i \leqslant N} \eta_{i} \leqslant r_{2}\right\}
$$

where $k_{1}+k_{2}+\cdots+k_{N}=m, 0 \leqslant k_{i} \leqslant r_{2}, 1 \leqslant i \leqslant N$.

As $r_{1}=0$, Theorem 2 implies

Corollary 1. Random variables $\eta_{1}^{\{\mathbf{2}\}}, \ldots, \eta_{\mathrm{N}}^{\{\mathbf{2}\}}$ are a generalized scheme of allocating $m$ particles in $N$ cells defined by the function $S_{0 r_{2}}(x)=\sum_{i=0}^{r_{2}} a_{i} x^{i}$.

Let $0 \leqslant r_{1}<\infty$. Consider random variables $\eta_{1}^{\{1\}}, \ldots, \eta_{\mathrm{N}}^{\{1\}}$ with the joint distribution

$$
\mathbf{P}\left\{\eta_{1}^{\{1\}}=k_{1}, \ldots, \eta_{\mathrm{N}}^{\{1\}}=k_{N}\right\}=\mathbf{P}\left\{\eta_{1}=k_{1}, \ldots, \eta_{\mathrm{N}}=k_{N} \mid r_{1} \leqslant \min _{1 \leqslant i \leqslant N} \eta_{i}\right\},
$$

where $k_{1}+k_{2}+\cdots+k_{N}=m, r_{1} \leqslant k_{i}, 1 \leqslant i \leqslant N$.

As $r_{2}=\infty$, Theorem 2 implies

Corollary 2. Random variables $\eta_{1}^{\{\mathbf{1}\}}, \ldots, \eta_{\mathrm{N}}^{\{\mathbf{1}\}}$ are a generalized scheme of allocating $m$ particles in $N$ cells defined by the function $S_{r_{1} \infty}(x)=\sum_{i=r_{1}}^{\infty} a_{i} x^{i}$.

We apply Theorem 1 and Theorem 2 to studying the asymptotic behavior of the probability of event $A_{n, N}\left(m, r, r_{1}, r_{2}\right)$ that each cell of each scheme contains at most $r$ particles if we know that each cell of each scheme contains at least $r_{1}$ and at most $r_{2}$ particles, that is, the probability of event $A_{n, N}(m, r)$ under the condition: each cell of each scheme contains at least $r_{1}$ and at most $r_{2}$ particles.

Theorem 3. Assume that $r_{1}<r<r_{2}, r<K<\infty$ and condition $\left(A_{r_{1}}\right)$ is satisfied. We denote $m_{1}=m-r_{1} N$. Then

$$
\mathbf{P}\left(A_{n, N}\left(m, r, r_{1}, r_{2}\right)\right)=\exp \left[\frac{-m_{1}\left(m_{1}-1\right) \cdots\left(m_{1}-\left(r-r_{1}\right)\right)}{N^{r-r_{1}-1}} \frac{a_{r+1}}{a_{r_{1}+1}^{r-r_{1}+1}} \alpha\left(1+O\left(\frac{1}{N}\right)\right)\right]
$$


uniformly in $r<m_{1}<K$ as $m, n, N \rightarrow \infty$ so that $\alpha=o\left(N^{r-r_{1}}\right)$. In particular, if $m, n, N \rightarrow$ $\infty$ so that $m_{1}$ is fixed, $m_{1}>r$ and $\frac{n}{N^{r-r_{1}}} \rightarrow \beta$, where $\beta<\infty$, then

$$
\mathbf{P}\left(A_{n, N}\left(m, r, r_{1}, r_{2}\right)\right) \rightarrow \exp \left[-m_{1}\left(m_{1}-1\right) \cdots\left(m_{1}-\left(r-r_{1}\right)\right) \frac{a_{r+1}}{a_{r_{1}+1}^{r-r_{1}+1}} \beta\right]
$$

Employing Corollary 1 of Theorem 2 instead of Theorem 2 in the proof of Theorem 3, we obtain

Corollary 1. Assume that $0<r<r_{2}$ and condition $\left(A_{0}\right)$ is satisfied. Then

$$
\mathbf{P}\left(A_{n, N}\left(m, r, 0, r_{2}\right)\right)=\exp \left[\frac{-(m)(m-1) \cdots(m-r)}{N^{r-1}} \frac{a_{r+1}}{a_{1}^{r+1}} \alpha\left(1+O\left(\frac{1}{N}\right)\right)\right],
$$

as $n, N \rightarrow \infty$ so that $\alpha=o\left(N^{r}\right)$. In particular, if $n, N \rightarrow \infty$ so that $\frac{n}{N^{r}} \rightarrow \beta$, where $\beta<\infty$, then

$$
\mathbf{P}\left(A_{n, N}\left(m, r, 0, r_{2}\right)\right) \rightarrow \exp \left[-m(m-1) \cdots(m-r) \frac{a_{r+1}}{a_{1}^{r+1}} \beta\right] .
$$

Remark 2. It follows from Theorem 3 and Corollary 1 of Theorem 3 that the asymptotic behavior of the probability of event $A_{n, N}\left(m, r, r_{1}, r_{2}\right)$ is independent of $r_{2}$.

Probability $\mathbf{P}\left(A_{n, N}(m, r)\right)$ has the following application in the theory of antinoise coding. Consider a code, which allows to fix at most $r$ replacement kind errors in a block. A particular case of such code is Hamming code (for Hamming code see, for instance, [10]). Suppose that we have $n$ messages. Each message has $N$ blocks and contains $m$ errors. We assume that the probability associated with different messages are independent and the errors are distributed in the blocks of the messages in accordance with some generalized allocating scheme. Interpreting errors as particles and cells as blocks, we observe that $\mathbf{P}\left(A_{n, N}(m, r)\right)$ is the probability of the even that all the errors in $n$ messages are fixed.

In work [1], the convergence of probability $\mathbf{P}\left(A_{n, N}(m, r)\right)$ was studied in the case of the scheme of allocating discernible particles in different cells. In [12], [13] there was studied the convergence of probability $\mathbf{P}\left(A_{n, N}(m, r)\right)$ in the general case. In works [14], [15], the convergence of probabilities of some analogues of events $A_{n, N}(m, r)$ was treated. As $r=1$ in [16] there was studied the convergence of event $A_{n, N}(m, r)$, in which the amount of particles in the blocks is random. The probabilities of events $A_{N}(m, r)$ in some analogues of generalized allocating scheme were studied in [17].

Let us consider the application of Theorem 1 to some schemes of probability combinatorics.

Random forests. A random forest having $N$ root and $m$ non-root vertices is a generalized scheme of allocating $m$ particles in $N$ cells with the function

$$
S(z)=\frac{1^{1-1}}{1 !} z+\frac{2^{2-1}}{2 !} z^{2}+\cdots \frac{r^{r-1}}{r !} z^{r}+\cdots,
$$

that is, it corresponds to the case $k=1$ (see [2]). This is the probability of the event that in $n$ random forests, each consisting of $N$ trees and $m$ non-root vertices, each tree has at most $r$ branches is equal

$\mathbf{P}\left(A_{n, N}(m, r)\right)=\exp \left[\frac{-(m-N)(m-N-1) \cdots(m-N-(r-1))(r+1)^{r-1}}{N^{r-2} r !} \alpha\left(1+O\left(\frac{1}{N}\right)\right)\right]$,

as $n, N \rightarrow \infty$ so that $\alpha=o\left(N^{r-1}\right)$. In particular, if $m, n, N \rightarrow \infty$ so that $m-N=m_{1}$ is fixed and $\frac{n}{N^{r-1}} \rightarrow \beta$, where $\beta<\infty$, then

$$
\mathbf{P}\left(A_{n, N}(m, r)\right) \rightarrow \exp \left[-\frac{m_{1}\left(m_{1}-1\right) \cdots\left(m_{1}-(r-1)\right)(r+1)^{r-1}}{r !} \beta\right] .
$$


Cycles in substitutions. A random substitution of degree $m$ containing exactly $N$ cycles is a generalized scheme of allocating $m$ particles in $N$ cells with the function $S(z)=-\ln (1-z)$ (see [2]). We note that $-\ln (1-z)=\sum_{k=1}^{\infty} \frac{1}{k} z^{k}$. This is why the probability of the event that in $n$ random substitutions, each being of degree $m$ and consisting of $N$ cycles, each cycle has the length at most $r$, is equal to

$$
\mathbf{P}\left(A_{n, N}(m, r)\right)=\exp \left[\frac{-(m-N)(m-N-1) \cdots(m-N-(r-1)) 2^{r}}{N^{r-2}(r+1)} \alpha\left(1+O\left(\frac{1}{N}\right)\right)\right],
$$

as $n, N \rightarrow \infty$ so that $\alpha=o\left(N^{r-1}\right)$. In particular, if $m, n, N \rightarrow \infty$ so that $m-N=m_{1}$ is fixed and $\frac{n}{N^{r-2}} \rightarrow \beta$, where $\beta<\infty$, then

$$
\mathbf{P}\left(A_{n, N}(m, r)\right) \rightarrow \exp \left[-\frac{m_{1}\left(m_{1}-1\right) \cdots\left(m_{1}-(r-1)\right) 2^{r}}{r+1} \beta\right] .
$$

Random partitions. An equiprobable partition of an integer positive number $m$ in $N$ ordered terms not exceeding $k$ is the generalized allocating scheme with the function

$$
S(z)=\frac{z^{k}}{1-z}=\sum_{i=k}^{\infty} z^{i}
$$

(see [2]), i.e., it satisfies condition $\left(A_{k}\right)$. This is why the probability of the event that in $n$ independent random partitions of an integer positive number $m$ into $N$ ordered terms not exceeding $k$, each element of the partition does not exceed $r$, is equal to

$$
\mathbf{P}\left(A_{n, N}(m, r)\right)=\exp \left[\frac{-(m-k N)(m-k N-1) \cdots(m-k N-(r-k))}{N^{r-k-1}} \alpha\left(1+O\left(\frac{1}{N}\right)\right)\right],
$$

as $m, n, N \rightarrow \infty$ so that $\alpha=o\left(N^{r-k}\right)$. In particular, if $m>r, n, N \rightarrow \infty$ so that $m-k N=m_{1}$ is fixed, $\frac{n}{N^{r-k}} \rightarrow \beta$, where $\beta<\infty$, then

$$
\mathbf{P}\left(A_{n, N}(m, r)\right) \rightarrow \exp \left[-m_{1}\left(m_{1}-1\right) \cdots\left(m_{1}-(r-k)\right) \beta\right] .
$$

\section{ProOfs}

Proof of Lemma A. We have

$$
\begin{aligned}
\mathbf{P}\left(A_{m N}(r)\right)= & \mathbf{P}\left\{\xi_{1} \leqslant r, \xi_{2} \leqslant r, \ldots \xi_{N} \leqslant r \mid \xi_{1}+\xi_{2}+\cdots+\xi_{N}=m\right\} \\
= & \frac{\mathbf{P}\left\{\xi_{1} \leqslant r, \xi_{2} \leqslant r, \ldots \xi_{N} \leqslant r, \xi_{1}+\xi_{2}+\ldots \xi_{N}=m\right\}}{\mathbf{P}\left\{\xi_{1}+\xi_{2}+\ldots \xi_{N}=m\right\}} \\
& =\frac{\left\{\left(k_{i}\right): k_{1}+k_{2}+\cdots+k_{N}=m, k_{i} \leqslant r, 1 \leqslant i \leqslant N\right\}}{\sum_{\left\{\left(k_{i}\right): k_{1}+k_{2}+\cdots+k_{N}=m\right\}} \mathbf{P}\left\{\xi_{1}=k_{1}\right\} \mathbf{P}\left\{\xi_{2}=k_{2}\right\} \mathbf{P}\left\{\xi_{2}=k_{2}\right\} \ldots \mathbf{P}\left\{\xi_{N}=k_{N}\right\}} \\
\sum_{\left\{\xi_{N}=k_{N}\right\}} & \frac{\left\{\left(k_{i}\right): k_{1}+k_{2}+\cdots+k_{N}=m, k_{i} \leqslant r, 1 \leqslant i \leqslant N\right\}}{\sum_{\left.\left\{k_{i}\right): k_{1}+k_{2}+\cdots+k_{N}=m\right\}} \frac{a_{k_{1}} x^{k_{1}}}{S(x)} \frac{a_{k_{2}} x^{k_{2}}}{S(x)} \ldots \frac{a_{k_{2}} x^{k_{2}}}{S(x)} \ldots \frac{a_{k_{N}} x^{k_{N}}}{S(x)}}
\end{aligned}
$$

The first identity in (1) is proven. Since

$$
a_{m}\left(S^{N}\right)=\frac{1}{2 \pi \mathrm{i}} \oint_{C} \frac{S(z)^{N}}{z^{m+1}} d z, \quad a_{m}\left(S_{r}^{N}\right)=\frac{1}{2 \pi \mathrm{i}} \oint_{C} \frac{S_{r}(z)^{N}}{z^{m+1}} d z
$$

the second identity in (1) holds true. The proof is complete. 
Proof of Theorem 1. By (1) the probability of event $A_{n, N}$ has the following representation

$$
\mathbf{P}\left(A_{n, N}\right)=\mathbf{P}\left(\cap_{i=1}^{n}\left\{\eta_{\mathrm{i} 1} \leqslant r, \ldots, \eta_{\mathrm{iN}} \leqslant r\right\}\right)=\left(\frac{\frac{1}{2 \pi \mathrm{i}} \oint_{C} \frac{S_{r}(z)^{N}}{z^{m+1}} d z}{\frac{1}{2 \pi \mathrm{i}} \oint_{C} \frac{S(z)^{N}}{z^{m+1}} d z}\right)^{n} .
$$

Hence, employing (5) and (2), where $m$ and $r$ are replaced by $m-k N$ and $r-k$, respectively, we obtain

$$
\begin{aligned}
\mathbf{P}\left(A_{n, N}(m, r)\right) & =\left(\frac{\frac{1}{2 \pi \mathrm{i}} \oint_{C} \frac{\left(\sum_{i=k}^{r} a_{i} z^{i}\right)^{N}}{z^{m+1}} d z}{\frac{1}{2 \pi \mathrm{i}} \oint_{C} \frac{\left(\sum_{i=k}^{\infty} a_{i} z^{i}\right)^{N}}{z^{m+1}} d z}=\left(\frac{\frac{1}{2 \pi \mathrm{i}} \oint_{C} \frac{\left(\sum_{i=0}^{r-k} a_{i+k} z^{i}\right)^{N}}{z^{m-k N+1}} d z}{\frac{1}{2 \pi \mathrm{i}} \oint_{C} \frac{\left(\sum_{i=0}^{\infty} a_{i+k} z^{2}\right)^{N}}{z^{m-k N+1}} d z}\right)^{n}\right. \\
& =\exp \left[\frac{-m_{1}\left(m_{1}-1\right) \cdots\left(m_{1}-(r-k)\right)}{N^{r-k-1}} \frac{a_{r+1}}{a_{k+1}^{r-k+1}} \alpha\left(1+O\left(\frac{1}{N}\right)\right)\right] .
\end{aligned}
$$

Estimate (3) is proven. It implies (4). This completes the proof of Theorem 1.

Proof of Theorem 2. We consider independent identically distributed random variables $\xi_{i}^{\prime}(x)$ with the distribution $\mathbf{P}\left(\xi_{i}^{\prime}(x)=j\right)=\frac{a_{j} x^{j}}{S_{r_{1} r_{2}}(x)}, r_{1} \leqslant j \leqslant r_{2}, \mathbf{P}\left(\xi_{i}^{\prime}(x)=j\right)=0, j \notin\left[r_{1}, r_{2}\right]$. Let $k_{1}+k_{2}+\cdots+k_{N}=m, r_{1} \leqslant k_{i} \leqslant r_{2}, 1 \leqslant i \leqslant N$. We have

$$
\begin{aligned}
& \mathbf{P}\left\{\eta_{1}^{\prime}=k_{1}, \ldots, \eta_{\mathrm{N}}^{\prime}=k_{N}\right\} \\
& =\frac{\mathbf{P}\left\{\xi_{1}=k_{1}, \ldots, \xi_{\mathrm{N}}=k_{N}, \xi_{1}+\cdots+\xi_{\mathrm{N}}=m, \xi_{i}=k_{i}, r_{1} \leqslant k_{i} \leqslant r_{2}, 1 \leqslant i \leqslant N\right\}}{\mathbf{P}\left\{\xi_{1}+\cdots+\xi_{\mathrm{N}}=m, \xi_{i}=k_{i}, r_{1} \leqslant k_{i} \leqslant r_{2}, 1 \leqslant i \leqslant N\right\}} \\
& =\frac{\mathbf{P}\left\{\xi_{1}=k_{1}, \ldots, \xi_{\mathrm{N}}=k_{N}\right\}}{\mathbf{P}\left\{\xi_{1}+\cdots+\xi_{\mathrm{N}}=m, \xi_{i}=k_{i}, r_{1} \leqslant k_{i} \leqslant r_{2}, 1 \leqslant i \leqslant N\right\}} \\
& =\frac{\prod_{i=1}^{N} \mathbf{P}\left\{\xi_{\mathrm{i}}=k_{i}\right\}}{\sum_{k_{1}+k_{2}+\cdots+k_{N}=m, r_{1} \leqslant k_{i} \leqslant r_{2}, 1 \leqslant i \leqslant N} \prod_{i=1}^{N} \mathbf{P}\left\{\xi_{i}=k_{i}\right\}} \\
& =\frac{\prod_{i=1}^{N} \frac{a_{k_{i}} x^{k_{i}}}{S(x)}}{\sum_{k_{1}+k_{2}+\cdots+k_{N}=m, r_{1} \leqslant k_{i} \leqslant r_{2}, 1 \leqslant i \leqslant N} \prod_{i=1}^{N} \frac{a_{k_{k}} x^{k_{i}}}{S(x)}}=\frac{\prod_{i=1}^{N} a_{k_{i}} x^{k_{i}}}{\sum_{k_{1}+k_{2}^{\prime}+\cdots+k_{N}=m, r_{1} \leqslant k_{i} \leqslant r_{2}, 1 \leqslant i \leqslant N} \prod_{i=1}^{N} a_{k_{i}} x^{k_{i}}} \\
& =\frac{\prod_{i=1}^{N} \frac{a_{k_{i}} x^{k_{i}}}{S_{r_{1} r_{2}}(x)}}{\sum_{k_{1}+k_{2}+\cdots+k_{N}=m, r_{1} \leqslant k_{i} \leqslant r_{2}, 1 \leqslant i \leqslant N} \prod_{i=1}^{N} \frac{a_{k_{i}} x^{k_{i}}}{S_{r_{1} r_{2}}(x)}}=\frac{\prod_{i=1}^{N} \mathbf{P}\left\{\xi_{\mathrm{i}}^{\prime}=k_{i}\right\}}{\mathbf{P}\left\{\xi_{1}^{\prime}+\cdots+\xi_{\mathrm{N}}^{\prime}=m\right\}} \\
& =\frac{\mathbf{P}\left\{\xi_{1}^{\prime}=k_{1}, \ldots, \xi_{\mathrm{N}}^{\prime}=k_{N}, \xi_{1}^{\prime}+\cdots+\xi_{\mathrm{N}}^{\prime}=m\right\}}{\mathbf{P}\left\{\xi_{1}+\cdots+\xi_{\mathrm{N}}^{\prime}=m\right\}} \\
& =\mathbf{P}\left\{\xi_{1}^{\prime}=k_{1}, \ldots, \xi_{\mathrm{N}}^{\prime}=k_{N}, \mid \xi_{1}^{\prime}+\cdots+\xi_{\mathrm{N}}^{\prime}=m\right\} .
\end{aligned}
$$

The proof is complete.

Proof of Corollary 1 of Theorem 2. Since

$$
\left\{\max _{1 \leqslant i \leqslant N} \eta_{i} \leqslant r_{2}\right\}=\left\{0 \leqslant \min _{1 \leqslant i \leqslant N} \eta_{i}, \max _{1 \leqslant i \leqslant N} \eta_{i} \leqslant r_{2}\right\},
$$


the distribution of random vector $\eta_{1}^{\{\mathbf{2}\}}, \ldots, \eta_{\mathrm{N}}^{\{\mathbf{2}\}}$ coincides with the distribution of random vector $\eta_{1}^{\prime}, \ldots, \eta_{\mathrm{N}}^{\prime}$ corresponding to the case $r_{1}=0$. Hence, we can apply Theorem 2 and this completes the proof.

Proof of Theorem 3. By Theorem 2 the identity

$$
\begin{aligned}
\mathbf{P}\left(A_{n, N}\left(m, r, r_{1}, r_{2}\right)\right) & =\left(\mathbf{P}\left\{\max _{1 \leqslant i \leqslant N} \eta_{i} \leqslant r \mid r_{1} \leqslant \min _{1 \leqslant i \leqslant N} \eta_{i}, \max _{1 \leqslant i \leqslant N} \eta_{i} \leqslant r_{2}\right\}\right)^{n} \\
& =\left(\mathbf{P}\left\{\max _{1 \leqslant i \leqslant N} \xi_{i}^{\prime} \leqslant r \mid \xi_{1}^{\prime}+\cdots+\xi_{N}^{\prime}=m\right\}\right)^{n}
\end{aligned}
$$

holds true. Applying Theorem 1 to the last expression in the above identity, we complete the proof.

\section{BIBLIOGRAPHY}

1. V.F. Kolchin. A class of limit theorems for conditional distributions. // Litov. Matem. Sborn. 8:1, 53-63 (1968). (in Russian).

2. V.F. Kolchin. Random graphs. Fizmatgiz, Moscow (2000). (in Russian).

3. A.N. Timashev. Asymptotic expansions in probability combinatorics. Scientific Publisher TVP, Moscow (2011). (in Russian).

4. A.N. Timashev. Generalized allocating scheme in the problems of probability combinatorics. Publishing House "Academy", Moscow (2011). (in Russian).

5. A.N. Timashev. Large deviations in probability combinatorics. Publishing House "Academy", Moscow (2011). (in Russian).

6. Yu.L. Pavlov. Random forests. Karelia Scientific Center of RAS, Petrozavodsk (1996). (in Russian).

7. A.V. Kolchin. On limit theorems for the generalised allocation scheme // Diskr. Matem. 15:4, 148-157 (2003). [Disc. Math. Appl. 13:6, 627-636 (2003).]

8. A.V. Kolchin, V.F. Kolchin. On transition of distributions of sums of independent identically distributed random variables from one lattice to another in the generalised allocation scheme // Diskr. Matem. 18:4, 113-127 (2006) [Disc. Math. Appl. 16:6, 527-540 (2006).]

9. A.V. Kolchin, V.F. Kolchin. On the transition of distributions of sums of random variables related to the generalised allocation scheme from one lattice to another // Diskr. Matem. 19:3, 15-21 (2007) [Disc. Math. Appl. 17:5, 455-461 (2007).]

10. F.A. Novikov. Discrete mathematics for programmers. Piter, St.-Petersburg (2004). (in Russian).

11. F.G. Avkhadiev and A.N. Chuprunov. The probability of a successful allocation of ball groups by boxes // Lobachevskii J. Math. 25, 3-7 (2007).

12. F.G. Avkhadiev, I.R. Kayumov, A.N. Chuprunov. Study of probability of successful allocation of particles in cells by the methods of complex analysis // Trudy Matem. Centra im. N.I. Lobachevskogo. 19, 6-7 (2003). (in Russian.)

13. I.R. Kayumov, A.N. Chuprunov. The probability of successful allocation of particles in cells (the general case) // Fundament. Prikl. Matem. 18, 119-128 (2013). [ J. Math. Sci. 209:1, 88-95 (2015).]

14. A.N. Chuprunov, B.I. Khamdeyev. On probabilistic aspects of error correction codes when the number of errors is a random set // Inform. Primen. 3:3, 52-59 (2009). (in Russian).

15. A.N. Chuprunov, B.I. Khamdeyev. The probability of correcting errors by an antinoise coding method when the number of errors belongs to a random set // Izv. Vyssh. Uchebn. Zaved. Mat. 8, 81-88 (2010). [Russ. Math. (Izv. VUZ. Matem.) 54:8, 67-73 (2010).]

16. A.N. Chuprunov, B.I. Khamdeev. On probability of correction of a random number of errors in an error-correcting coding // Diskr. Matem. 22:2, 41-50 (2010). [Disc. Math. Appl. 20:2, 179-190 (2010).]

17. E.V. Khvorostyanskaya, Y.L. Pavlov. Limit distributions of the maximum filling of cells in one allocation scheme // European Researcher. 76:6-1, 1019-1027 (2014). (in Russian). 
Afonina Alexandra Igorevna,

Kazan Federal University,

Lobachevskii Institute of Mathematics and Mechanics, Kremlevskaya str. 35,

420008, Kazan, Russia

E-mail: sanyagirl89@mail.ru

Kayumov Il'giz Rifatovich,

Kazan Federal University,

Lobachevskii Institute of Mathematics and Mechanics,

Kremlevskaya str. 35,

420008, Kazan, Russia

E-mail: ikayumov@kpfu.ru

Chuprunov Alexei Nikolaevich,

Kazan Federal University,

Lobachevskii Institute of Mathematics and Mechanics,

Kremlevskaya str. 35,

420008, Kazan, Russia

E-mail: Alexey.Chuprunov@ksu.ru 